\title{
The Value of Trabecular Bone Score in the Evaluation of Bone Quality in a Patient with Ankylosing Spondylitis
}

\author{
Mozhdeh Zabihiyeganeh, ${ }^{1}$ and Alireza Mirzaei ${ }^{1,{ }^{*}}$ \\ ${ }^{1}$ Bone and Joint Reconstruction Research Center, Shafa Orthopedic Hospital, Iran University of Medical Sciences, Tehran, Iran \\ "Corresponding author: Alireza Mirzaei, Shafa Orthopedic Hospital, Baharestan Sq, Tehran, Iran. Tel: +98-2133542000-8, Fax: +98-2133542020, E-mail: \\ mirzaeialireza26@gmail.com
}

Received 2017 March 13; Revised 2017 April 19; Accepted 2017 July 06.

Keywords: Areal Bone Mineral Density, Trabecular Bone Score, Ankylosing Spondylitis, Osteoporosis

\section{Dear Editor,}

Patients with ankylosing spondylitis (AS) have an increased risk of developing osteoporosis, mainly caused by systemic inflammation and decreased mobility(1). For this reason, patients with AS demonstrate an increased risk of fractures, especially vertebral fractures, in comparison with the general population (2). However, measurements of areal bone mineral density (aBMD) are overestimated and unreliable in AS patients, because of the presence of osteo-proliferation and syndesmophytes. Significantly increased aBMD measures have been reported in patients with advanced AS (3). Consequently, the diagnosis of osteoporosis could be difficult in affected patients (1).

Considering bone microarchitecture as another determinant of bone strength, and regarding less impact of osteoproliferation on bone microarchitecture, it could be a better predictor of bone strength in these patients (4). Trabecular bone score (TBS), a new noninvasive tool for the measurement of bone microarchitecture, could be used complementary to aBMD in the evaluation of bone quality in AS patients (5). In this letter, the authors discuss the value of TBS in the evaluation of bone quality in a patient with AS, and discuss its advantages over aBMD.

A 47-year-old male with a 10-year history of inflammatory back pain was referred to the researcher's center. Acute phase reactants including erythrocyte sedimentation rate (ESR) and C-reactive protein (CRP) were elevated and positive HLA-B27 was found. Other laboratory tests were within the normal range. Typical syndesmophytes were also observed in the spinal X-rays of the patient. The diagnosis of AS was made according to the assessment of spondylo arthritis international society (ASAS) criteria. Subsequently, the therapeutic approach was started with an anti-inflammatory dose of indomethacin. However, considering the patient's unresponsiveness, infliximab was added to his therapeutic regimen.

Given the 10-year history of the disease, osteoporosis evaluation was indicated for the patient and aBMD was ordered. Considering the normal aBMD results of the patient (T-score $>-1$ ), according to the world health organization (WHO) criteria, no osteoporosis treatment was indicated for the patient. However, since the aBMD results was probably artifactually elevated by the presence of typical syndesmophytes, the patients was asked to recheck the aBMD results in another center, which was equipped to the TBS software. While the aBMD results of the second exam were the same as the first one, the patient's bone microarchitecture was classified as fully degraded (TBS $\leq 1.20$ ). Consequently, zoledronic acid (Aclasta) was administered as the osteoporosis therapeutic agent.

The case showed that regarding the presence of sindesmophytes, aBMD is not a reliable marker for the evaluation of bone quality in the affected patients (1). Considering the presence of typical syndesmophytes in the X-rays of the patient's vertebrae, the authors concluded that the aBMD is probably overestimated, and TBS could be more helpful in the evaluation of bone strength in this patient. The fully degraded bone microarchitecture of the patient evaluated by TBS confirmed the hypothesis, and the low bone quality of the patient was managed according to TBS and not aBMD results.

Based on our observation, the value of TBS could be more emphasized in the evaluation of bone quality, especially in AS patients with normal or increased aBMD. Such deduction has also been reported in other investigations. Wildberger et al. evaluated the impact of lumbar syndesmophytes on aBMD and TBS in males with axial spondyloarthritis. Their results suggested that TBS is not influenced by the syndesmophytes opposite to spine aBMD, and could be measured in this population in addition to the aBMD to assess bone fragility (4).

Paolino et al. also evaluated bone microarchitectural status of the lumbar spine in patients with AS. Their results showed increased aBMD and decreased TBS measures 
in these patients, reflecting the value of TBS in providing skeletal information that are not captured from aBMD measurement, at least in patients with AS with vertebral fractures (5).

In addition, management of osteoporosis and prevention of osteoporotic fracture in patients with AS with low bone quality needs further clarifications in large cohort studies. To the best of the authors' knowledge, there was no clear guideline for the management of low bone quality in patients with AS and normal aBMD. Available clinical practice guidelines suggest non-pharmacological (i.e. general exercise program) and pharmacological treatments (i.e., vitamin D, bisphosphonates) (6).

A growing body of evidence has shown that different osteoporosis drugs effect different characteristics of the bone (7). For instance, Triparatide is reported to be most effective if bone microarchitecture is deteriorated, while Bisphosphonates is regarded as the first line of treatment in osteoporotic patients with normal TBS (7). The unanswered questions are whether therapeutic intervention should be considered in cases with deteriorated TBS and artifactually normal BMD, and which therapeutic agent should be administered. The answer of these questions remains to be explored by future investigations.

Moreover, although anti-TNF agents increase BMD, there is no evidence regarding their effect on the osteoporotic fracture reduction. However, we have a low thresh- old to start biologic therapy in patients noted to have low bone quality and active AS.

\section{References}

1. Cooper C, Carbone L, Michet CJ, Atkinson EJ, O'Fallon WM, Melton L3. Fracture risk in patients with ankylosing spondylitis: a population based study. J Rheumatol. 1994;21(10):1877-82. [PubMed: 7837154].

2. El Maghraoui A. Osteoporosis and ankylosing spondylitis. Joint Bone Spine. 2004;71(4):291-5. doi: 10.1016/j.jbspin.2003.06.002. [PubMed: 15288853].

3. Mullaji AB, Upadhyay SS, Ho EK. Bone mineral density in ankylosing spondylitis. DEXA comparison of control subjects with mild and advanced cases. J Bone Joint Surg Br. 1994;76(4):660-5. [PubMed: 8027159].

4. Wildberger L, Boyadzhieva V, Hans D, Stoilov N, Rashkov R, AubryRozier B. Impact of lumbar syndesmophyte on bone health as assessed by bone density (BMD) and bone texture (TBS) in men with axial spondyloarthritis. Joint Bone Spine. 2017;84(4):463-6. doi: 10.1016/j.jbspin.2016.05.015. [PubMed: 27450198].

5. Paolino S, Botticella G, Casabella A, Fasciolo D, Sulli A, Cutolo M. Fri0232 evaluations of bone microarchitectural status of the lumbar spine in ankylosing spondylitis patients by trabecular bone score assessment. Ann Rheum Dis. 2015;74(2):508-9.

6. Hinze AM, Louie GH. Osteoporosis Management in Ankylosing Spondylitis. Curr Treatm Opt Rheumatol. 2016;2(4):271-82. doi: 10.1007/s40674-016-0055-6. [PubMed: 28620575].

7. Senn C, Gunther B, Popp AW, Perrelet R, Hans D, Lippuner K. Comparative effects of teriparatide and ibandronate on spine bone mineral density (BMD) and microarchitecture (TBS) in postmenopausal women with osteoporosis: a 2-year open-label study. Osteoporos Int. 2014;25(7):1945-51. doi: 10.1007/s00198-014-2703-8. [PubMed: 24760244]. 